Article

\title{
Influence of the Azulene Ring on the Enantioseparation of 1,5-Diols
}

\author{
Dana A. Horgen and Charles M. Garner * \\ Department of Chemistry and Biochemistry, Baylor University, Waco, TX 76798, USA; \\ E-Mail: dana_horgen@baylor.edu \\ * Author to whom correspondence should be addressed; E-Mail: Charles_Garner@baylor.edu; \\ Tel.: +1-254-710-6862; Fax: +1-254-710-4272.
}

Received: 3 April 2014; in revised form: 9 May 2014 / Accepted: 12 May 2014 /

Published: 16 May 2014

\begin{abstract}
The enantioseparation of a series of six azulene-centered 1,5-diol enantiomers was studied employing two cellulose-based chiral stationary phases under normal phase conditions (isopropanol/hexanes). The separations were generally quite good on Chiralcel-OD-H, with $\alpha$ values ranging from 1.2 to 8.4 (average 4.0) and resolution values of 0.4-8.3 (average 4.7). Only one of the six enantiomer pairs was not well resolved, but was well separated on Lux cellulose $2\left(\alpha 1.4, \mathrm{R}_{\mathrm{s}} 8.7\right)$. It was observed that the enantioseparations of the $\mathrm{RS} / \mathrm{SR}$ diastereomers (ave $\alpha=7.8, \mathrm{R}_{\mathrm{s}}=8.2$ ) were dramatically better than that of the corresponding RR/SS diastereomers (ave $\alpha=2.1, \mathrm{R}_{\mathrm{s}}=3.0$ ) on Chiralcel-OD-H. The better-resolved diastereomer pairs correspond to the more strongly retained diastereomers on silica gel. The enantiomers of two benzene 1,5-diols were much more poorly separated on both stationary phases, suggesting that the unusual polarity of the azulene ring enhances critical interactions with these phases.
\end{abstract}

Keywords: chiral azulene; chiral high-pressure liquid chromatography; enantioseparation

\section{Introduction}

Over the past two decades, the ability to accomplish enantiomer separations chromatographically has become an extremely valuable analytical tool and, to a lesser extent, preparatively useful [1,2]. There are many important applications, particularly with respect to pharmaceuticals, ranging from quality control in production to monitoring the environmental fate of these materials $[3,4]$. 
Azulene is isomeric with the relatively common naphthalene ring, yet is dramatically different in some of its properties. Azulenes are almost unique among hydrocarbons in having large $(\sim 1 \mathrm{D})$ dipole moments [5], roughly equivalent to that of $\mathrm{HCl}$ and over half that of water. They also represent one of the smallest conjugated systems having a visible chromophore; azulenes are typically blue or purple, though derivatives can be of nearly any color [6]. The azulene ring has been incorporated into a variety of structures, including pharmaceuticals (sodium azulene sulfonate) [7]. Remarkably, however, very few chiral azulene derivatives have been reported in the literature [8-13], and only one where the enantiomers were separated chromatographically [13].

During studies of chromatographic separations of a series of racemic azulene 1,5-diols (1-6, Figure 1), we found [14] that the diastereomers were remarkably well separated $\left(\Delta \mathrm{R}_{\mathrm{f}} \sim 0.22-0.46\right)$ on silica gel TLC. Subsequently, the chromatographic enantiomer separation using chiral High Performance Liquid Chromatography (HPLC) was undertaken partly to determine if the azulene ring was advantageous in cellulose-cased enantiomer separations. The results of this study are reported here.

Figure 1. Azulene-1,5-diols employed in this study (all racemic).
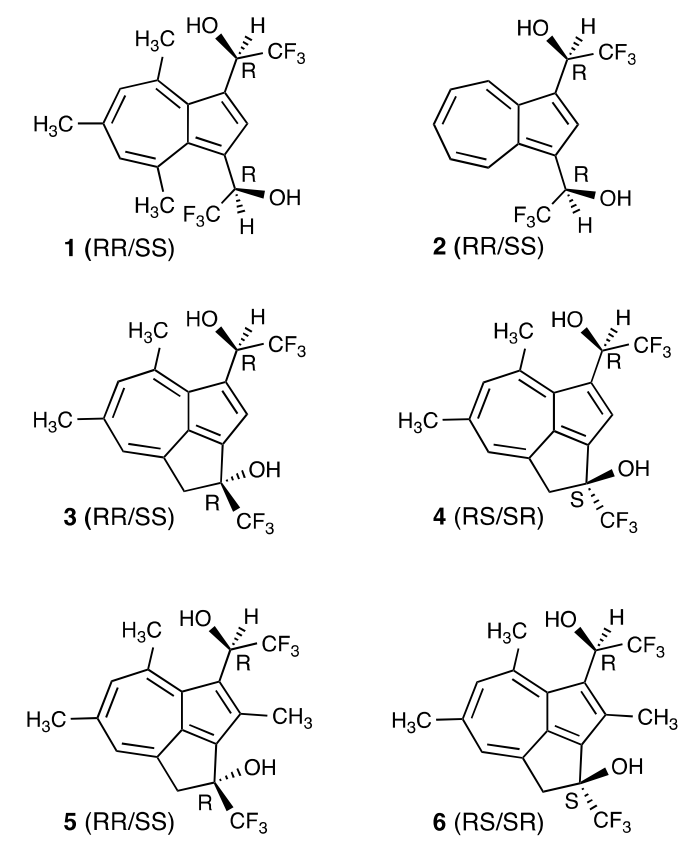

\section{Experimental Section}

\subsection{Instrumentation}

High Performance Liquid Chromatography (HPLC) was run at ambient temperature using a Beckman System Gold 126 Solvent Module instrument with a $5 \mu \mathrm{m}$ Chiralcel OD-H column, $2.1 \mathrm{~mm}$ ID $\times 150 \mathrm{~mm}$, and a $5 \mu \mathrm{m}$ Phenomonex Lux Cellulose 2 column, $4.6 \mathrm{~mm}$ ID $\times 150 \mathrm{~mm}$ (with SecurityGuard $^{\mathrm{TM}}$ precolumn filter, $3 \mathrm{~mm} \mathrm{ID} \times 4 \mathrm{~mm}$ ), with isopropanol (IPA) and hexanes (Hex) as the mobile phase, with a Beckman System Gold 168 UV Detector.

Ultraviolet (UV) spectroscopic data was collected utilizing a Shimadzu UV-2550 spectrometer over a wavelength range of 400 to $200 \mathrm{~nm}$. Circular Dichroism (CD) spectra were collected using a JASCO 810 spectrometer over a wavelength range of 400 to $200 \mathrm{~nm}$. 


\subsection{Example Procedure for Separation and Analysis of Enantiomers, $\boldsymbol{1}_{R S / S R}$}

Sample for HPLC was prepared by dissolving $3.8 \mathrm{mg}$ of the racemic azulene diol into $1.5 \mathrm{~mL}$ of $10 \%$ IPA:Hex. Then $20 \mu \mathrm{L}$ of the solution was injected into the HPLC utilizing the $5 \mu \mathrm{m}$ Phenomenex Lux cellulose 2 column with the column pressure maximum at 2 atm and a flow rate of $1.0 \mathrm{~mL} / \mathrm{min}$ at ambient temperature. The eluent solution gradient began at a concentration of 5\% IPA:Hex and was increased to $20 \%$ IPA:Hex evenly over $20 \mathrm{~min}$. The enantiomers eluted at $5.9 \mathrm{~min}$ for the faster, and $7.92 \mathrm{~min}$ for the slower. UV spectra were gathered by taking the collected fractions and diluting them with methanol $(\mathrm{MeOH})$. To obtain $\mathrm{CD}$ spectra the IPA:Hex was removed under vacuum and the compounds were dissolved in $\mathrm{MeOH}$. First a blank of pure $\mathrm{MeOH}$ was run in a $1 \mathrm{~mm}$ quartz cuvette from 195 to $400 \mathrm{~nm}$ (CD parameters: sensitivity high (5 mdeg), data pitch 0.1, scanning mode: continuous, scanning speed: $100 \mathrm{~nm} / \mathrm{min}$, response $0.25 \mathrm{~s}$, band width: $1 \mathrm{~nm}$, accumulation 5 , room temperature). The Cotton Effect observed for the enantiomers was greatest at $349.8 \mathrm{~nm}$ with 11.64 for the faster and -6.47 for the slower eluting enantiomers, respectively.

\section{Results and Discussion}

The synthesis of diols 1-6 has been recently reported [14]. These diols were formed as mixtures of diastereomers (RR/SS vs. RS/SR) that were easily separated by silica gel chromatography. All of these are chiral compounds except for the achiral meso $(\mathrm{RS}=\mathrm{SR})$ diastereomers of diols $\mathbf{1}$ and 2 (not shown). Note that diols $\mathbf{3 / 4}$, and $\mathbf{5 / 6}$ are diastereomers of one another. We now find that under normal-phase conditions (IPA in hexanes) Chiralcel-OD-H (cellulose-tri-(3,5-dimethyl phenyl) carbamate) coated on $5 \mu \mathrm{m}$ silica gel gave baseline resolutions $\left(\mathrm{R}_{\mathrm{s}} \sim 3-8\right)$ for five $(\mathbf{2}-\mathbf{6})$ of the six pairs of enantiomers (Table 1, examples shown in Figures 2 and 3).

Table 1. Summary of chiral High Performance Liquid Chromatography (HPLC) data for each of the enantiomers. Samples were eluted with isopropanol (IPA):hexanes (Hex) on a Chiralcel OD-H column.

\begin{tabular}{ccccccccc}
\hline Compound & $\begin{array}{c}\mathbf{R}_{\mathbf{f}} \\
\text { Value }\end{array}$ & $\begin{array}{c}\text { Rt 1 } \\
(\mathbf{m i n})\end{array}$ & $\begin{array}{c}\text { Rt 2 } \\
(\mathbf{m i n})\end{array}$ & $\begin{array}{c}\mathbf{w}_{\mathbf{1} / \mathbf{2}} \mathbf{1} \\
(\mathbf{m i n})\end{array}$ & $\begin{array}{c}\mathbf{w}_{\mathbf{1} / \mathbf{2}} \mathbf{2} \\
(\mathbf{m i n})\end{array}$ & Dead Volume & $\mathbf{R}_{\mathbf{s}}$ & $\boldsymbol{\alpha}$ \\
\hline $\mathbf{1}$ & 0.59 & $1.43^{\mathrm{f}}$ & $1.61^{\mathrm{f}}$ & 0.30 & 0.30 & $0.41 \mathrm{~mL}$ & 0.35 & 1.17 \\
$\mathbf{2}$ & 0.39 & $4.70^{\mathrm{e}}$ & $9.80^{\mathrm{e}}$ & 0.41 & 0.90 & 0.38 & 4.61 & 2.18 \\
$\mathbf{3}$ & 0.63 & $1.75^{\mathrm{f}}$ & $2.97^{\mathrm{f}}$ & 0.16 & 0.31 & 0.41 & 3.10 & 1.91 \\
$\mathbf{4}$ & 0.40 & $1.30^{\mathrm{g}}$ & $8.13^{\mathrm{g}}$ & 0.21 & 0.76 & 0.38 & 8.28 & 8.43 \\
$\mathbf{5}$ & 0.58 & $1.88^{\mathrm{b}}$ & $4.73^{\mathrm{b}}$ & 0.27 & 0.63 & 0.40 & 3.74 & 2.93 \\
$\mathbf{6}$ & 0.38 & $1.87^{\mathrm{c}}$ & $11.15^{\mathrm{c}}$ & 0.39 & 0.97 & 0.38 & 8.05 & 7.25 \\
$\mathbf{7}$ & 0.31 & $6.92^{\mathrm{b}}$ & $7.68^{\mathrm{b}}$ & 0.70 & 1.14 & 0.46 & 0.49 & 1.12 \\
$\mathbf{8}$ & 0.10 & $2.78^{\mathrm{a}}$ & $3.82^{\mathrm{a}}$ & 0.16 & 0.30 & 0.80 & 2.65 & 1.53 \\
\hline
\end{tabular}

${ }^{*} \mathrm{R}_{\mathrm{f}}$ values published in Horgen et al. 2014 [14]. Solvent gradients: ${ }^{a}$ isocratic $5 \%$ IPA in hexanes; ${ }^{b} 5 \%$ to $10 \%$ IPA in hexanes over $22 \mathrm{~min} ;{ }^{\mathrm{c}} 10 \%$ to $20 \%$ IPA in hexanes over $22 \mathrm{~min}$; ${ }^{\mathrm{d}}$ isocratic $20 \%$ IPA in hexanes; ${ }^{\mathrm{e}} 7.5 \%$ to $10 \%$ IPA in hexanes over $22 \mathrm{~min} ;{ }^{\mathrm{f}} 2.75 \%$ to $5 \%$ IPA in hexanes over $22 \mathrm{~min} ;{ }^{\mathrm{g}} 10 \%$ to $15 \%$ IPA in hexanes over $22 \mathrm{~min}$. 
Figure 2. Separation of racemic compound 2 on Chiralcel-OD-H (solvent: $7.5 \%$ IPA in hexanes to $10 \%$ IPA over $22 \mathrm{~min}$ ), representing an average separation on this column.

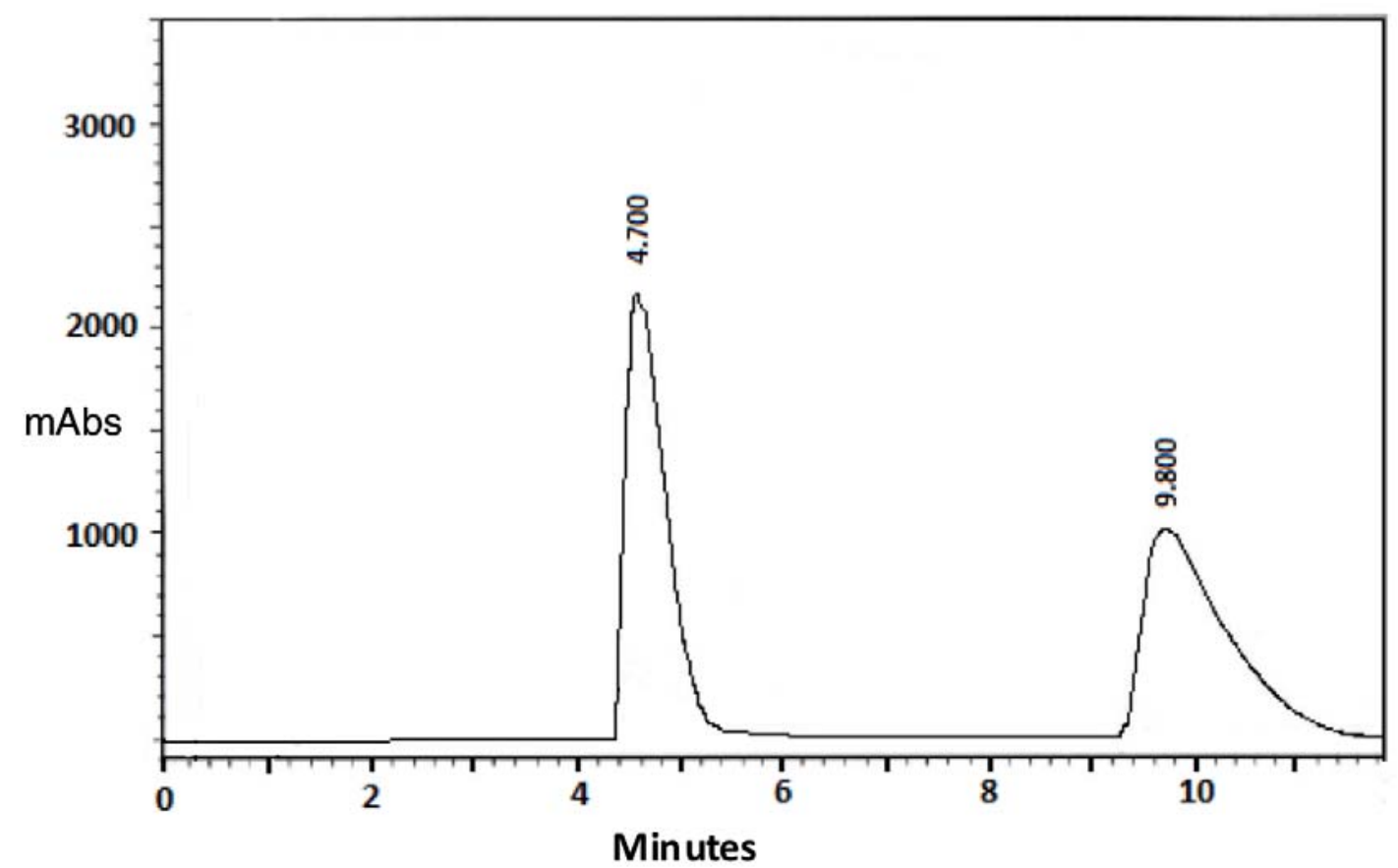

Figure 3. HPLC for compound 1 on the Chiralcel OD-H column using isocratic 5\% IPA in hexanes.

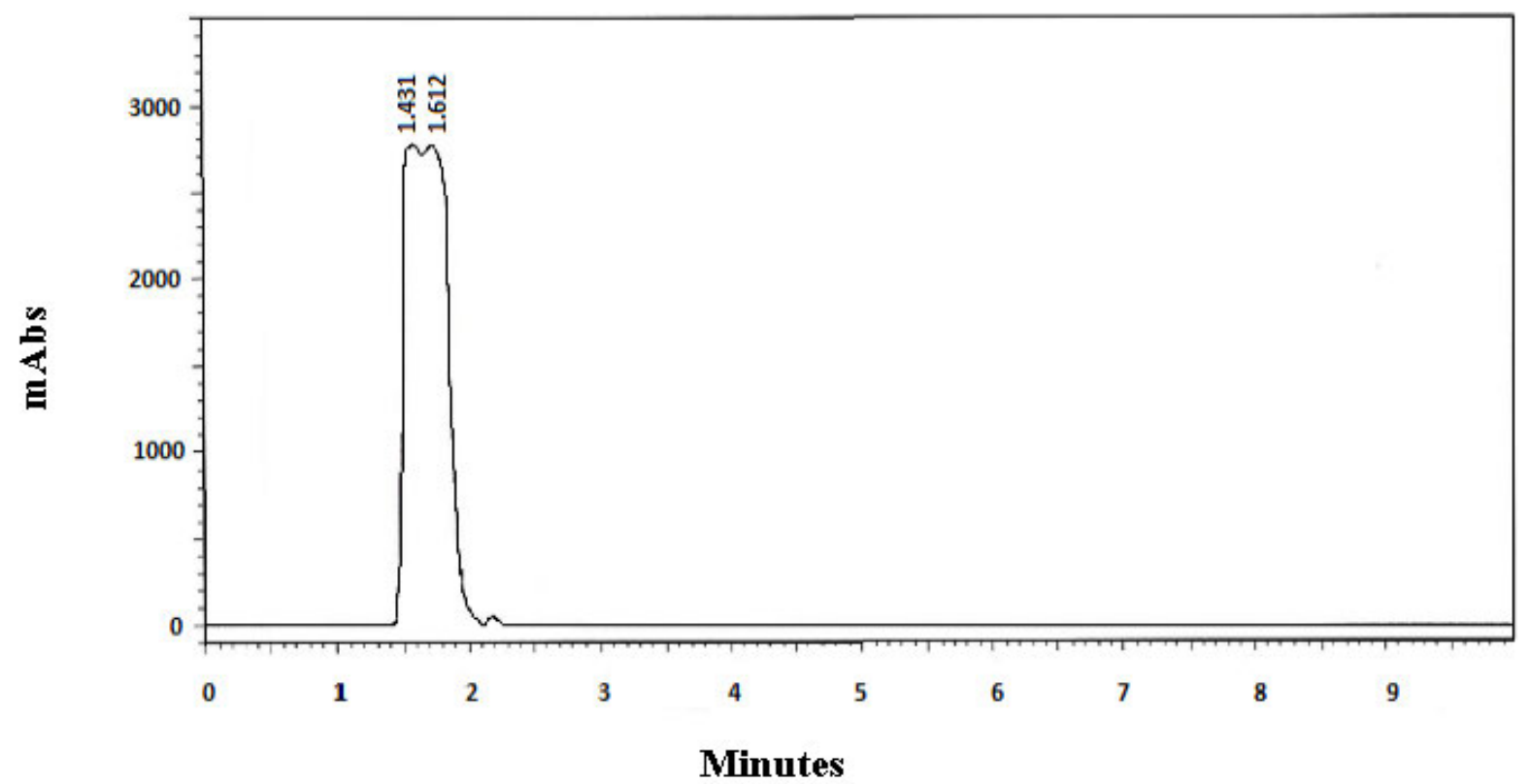

A chromatogram with typical resolution is shown in Figure 2. The remaining diol (1) enantiomers were resolved $\left(R_{s} 8.7\right)$ by the Phenomenex Lux cellulose 2 column (Table 2, example shown in Figure 4), again under normal-phase conditions. Three of these compounds (1, 2 and 4) were screened on other chiral columns and under various conditions; these data are given in Table 3. 
Table 2. Results from compounds run on a Phenomonex Lux Cellulose 2 column eluted with IPA in hexanes.

\begin{tabular}{ccccccccc}
\hline Compound & $\begin{array}{c}\mathbf{R}_{\mathbf{f}} \\
\text { value }^{*}\end{array}$ & $\begin{array}{c}\mathbf{R t ~ 1} \\
(\mathbf{m i n})\end{array}$ & $\begin{array}{c}\mathbf{R t ~ 2} \\
(\mathbf{m i n})\end{array}$ & $\begin{array}{c}\mathbf{w}_{\mathbf{1} / \mathbf{2}} \mathbf{1} \\
(\mathbf{m i n})\end{array}$ & $\begin{array}{c}\mathbf{w}_{\mathbf{1} / \mathbf{2}} \mathbf{2} \\
(\mathbf{m i n})\end{array}$ & $\begin{array}{c}\text { Dead } \\
\text { Volume }\end{array}$ & $\mathbf{R}_{\mathbf{s}}$ & $\boldsymbol{\alpha}$ \\
\hline $\mathbf{1}$ & 0.59 & $5.90^{\mathrm{d}}$ & $7.92^{\mathrm{d}}$ & 0.16 & 0.19 & $1.14 \mathrm{~mL}$ & 8.71 & 1.42 \\
$\mathbf{2}$ & 0.39 & $3.23^{\mathrm{c}}$ & $3.53^{\mathrm{c}}$ & 0.46 & 0.46 & 1.13 & 0.38 & 1.14 \\
$\mathbf{3}$ & 0.63 & $5.32^{\mathrm{b}}$ & $6.10^{\mathrm{b}}$ & 0.21 & 0.24 & 1.25 & 2.06 & 1.19 \\
$\mathbf{4}$ & 0.40 & $2.28^{\mathrm{c}}$ & $2.85^{\mathrm{c}}$ & 0.55 & 0.55 & 1.15 & 0.61 & 1.50 \\
$\mathbf{5}$ & 0.58 & $3.82^{\mathrm{b}}$ & $4.93^{\mathrm{b}}$ & 0.19 & 0.25 & 1.45 & 2.98 & 1.47 \\
$\mathbf{6}$ & 0.38 & $6.65^{\mathrm{b}}$ & $7.77^{\mathrm{b}}$ & 0.36 & 0.38 & 1.40 & 1.78 & 1.21 \\
$\mathbf{7}$ & 0.31 & $3.75^{\mathrm{b}}$ & $3.98^{\mathrm{b}}$ & 0.12 & 0.24 & 1.27 & 0.76 & 1.09 \\
$\mathbf{8}$ & 0.10 & $9.70^{\mathrm{b}}$ & $10.4^{\mathrm{b}}$ & 0.51 & 0.70 & 1.20 & 0.63 & 1.08 \\
\hline
\end{tabular}

${ }^{*} \mathrm{R}_{\mathrm{f}}$ values published in the Horgen et al. 2014 [14]. Solvent gradients: ${ }^{\mathrm{a}}$ isocratic 5\% IPA in hexanes; ${ }^{\mathrm{b}} 5 \%$ to $10 \%$ IPA in hexanes over $22 \mathrm{~min} ;{ }^{\mathrm{c}} 10 \%$ to $20 \%$ IPA in hexanes over $22 \mathrm{~min} ;{ }^{\mathrm{d}}$ isocratic $20 \%$ IPA in hexanes; ${ }^{\mathrm{e}} 7.5 \%$ to $10 \%$ IPA in hexanes over $22 \mathrm{~min} .{ }^{\mathrm{f}} 2.75 \%$ to $5 \%$ IPA in hexanes over $22 \mathrm{~min} .{ }^{\mathrm{g}} 10 \%$ to $15 \%$ IPA in hexanes over $22 \mathrm{~min}$.

Figure 4. HPLC for compound 1 on the Lux column using isocratic 20\% IPA in hexanes.

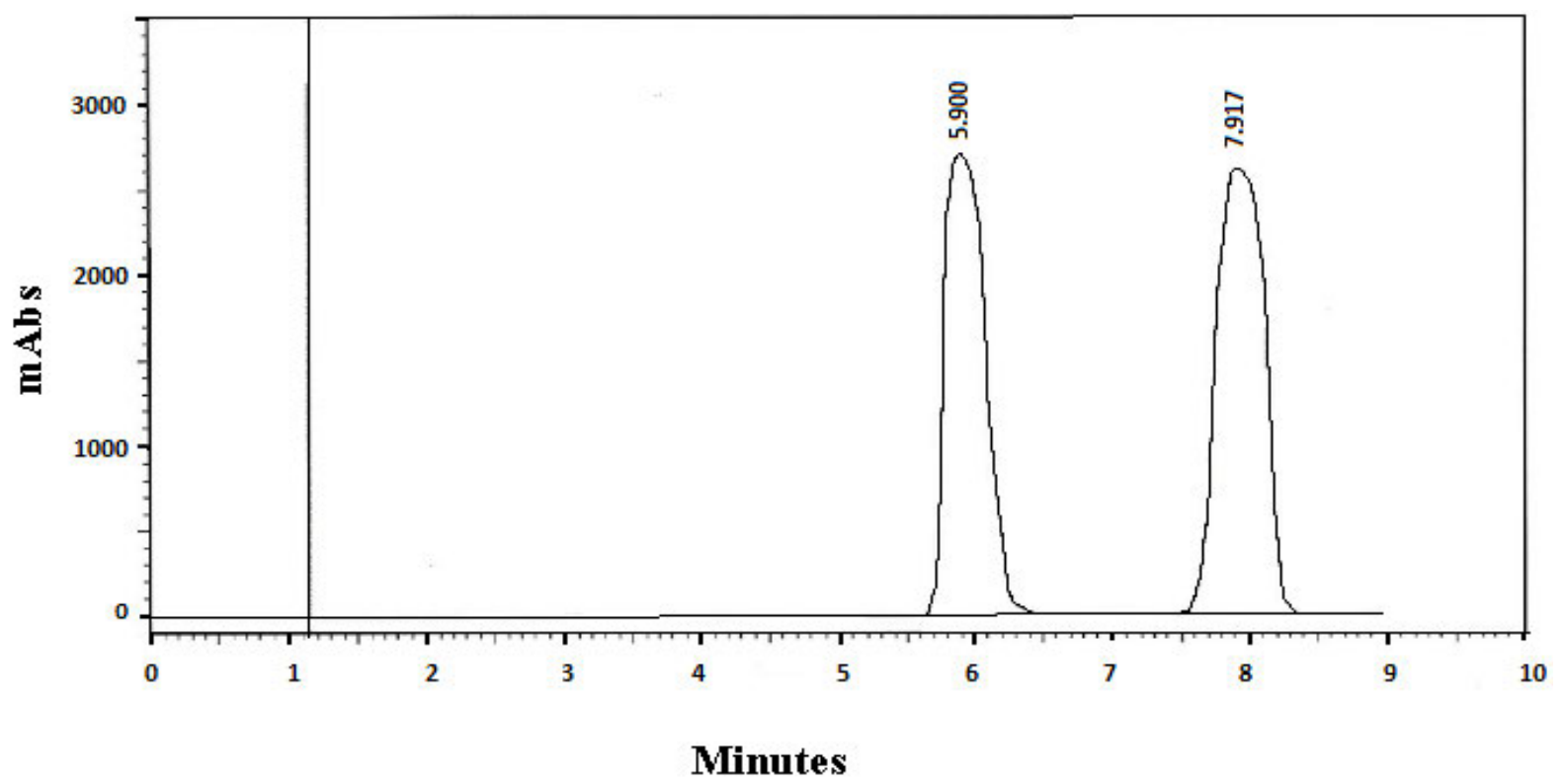

For comparison, the closely related benzene compounds 7 and 8 (Figure 5) were prepared by known $[15,16]$ methods to study the enantioseparation of the azulene ring relative to a phenyl ring. These benzene diols consistently exhibited inferior resolution relative to the azulene diols, particularly on Chiralcel-OD-H. The average $\alpha$ value for the azulene diols was 3.98 (Chiralcel-OD-H) and 1.32 (Lux cellulose 2), while the benzene diols averaged 1.33 and 1.09 on the same phases, respectively. 
Table 3. Results from compounds screened by Phenomenex, Regis Technologies and Chiral Technologies on various columns with both reverse and normal mobile phases.

\begin{tabular}{|c|c|c|c|c|c|}
\hline Compound & Column Name & Stationary Phase & Solvent System & $\mathbf{R}_{\mathbf{s}}$ & $\alpha$ \\
\hline 1 & Phenomenex Lux 5u Cellulose 4 & Cellulose tris-(4-chloro-3-methylphenylcarbamate) & 60:40:0.1 AmmBi:ACN:DEA ${ }^{\mathrm{c}}$ & 4.40 & 1.23 \\
\hline 1 & Phenomenex Lux 5u Cellulose 3 & Cellulose tris-(4-methylbenzoate & 60:40:0.1 AmmBi:ACN:DEA ${ }^{c}$ & 2.90 & 1.21 \\
\hline 1 & Phenomenex Lux 5u Cellulose 2 & Cellulose tris-(3-chloro-4-methylphenylcarbamate) & 60:40:0.1 AmmBi:ACN:DEA ${ }^{\mathrm{c}}$ & 1.03 & 1.14 \\
\hline 1 & Phenomenex Lux 5u Amylose 2 & Amylose tris-(5-chloro-2-methylphenylcarbamate) & 60:40:0.1 AmmBi:ACN:DEA ${ }^{\mathrm{c}}$ & 0.82 & 1.07 \\
\hline 1 & Phenomenex Lux 5u Cellulose 2 & Cellulose tris-(3-chloro-4-methylphenylcarbamate) & 90:10:0.1 Hex:IPA:DEA ${ }^{\mathrm{c}}$ & 4.45 & 1.52 \\
\hline 1 & Phenomenex Lux 5u Amylose 2 & Amylose tris-(5-chloro-2-methylphenylcarbamate) & 90:10:0.1 Hex:IPA:DEA ${ }^{\mathrm{c}}$ & 3.20 & 1.80 \\
\hline 2 & (S,S)-Whelk-O 1 (Regis Tech.) & 4-(3,5-dinitrobenzamido) tetrahydrophenathrene on silica & 90:10 Hex:IPA & 4.22 & 1.23 \\
\hline 2 & RegisPack $^{\mathrm{a}}$ & Tris-(3,5-dimethylphenyl) carbamoyl amylose & 90:10 Hex:MeOH & 4.64 & 1.22 \\
\hline 2 & RegisCell ${ }^{\mathrm{b}}$ & Tris-(3,5-dimethylphenyl) carbamoyl cellulose & $85: 15 \mathrm{Hex}^{\mathrm{EtOH}}{ }^{\mathrm{c}}$ & 9.51 & 1.78 \\
\hline 4 & CHIRALPAK IB-3 (Chiral Tech.) & Cellulose tris-(3,5-dimethylphenylcarbamate) & 80:20 Hex:EtOH ${ }^{\mathrm{c}}$ & 10.64 & 1.31 \\
\hline
\end{tabular}

${ }^{a}$ Same stationary phase as the ChiralPack AD.; ${ }^{\mathrm{b}}$ Same stationary phase as the Chiralcel OD; ${ }^{\mathrm{c}}$ The acronyms used above represent the following: ammonium bicarbonate (AmmiBi), acetonitrile (ACN), diethanolamine (DEA), ethanol (EtOH).

Figure 5. Benzene diols and benzene and naphthalene mono-alcohols for comparison.

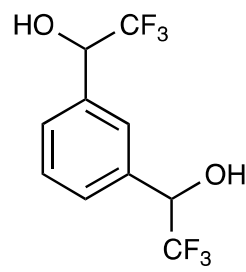

7

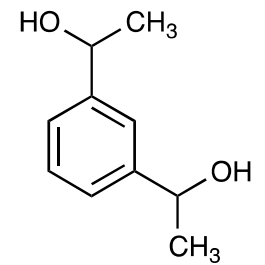

8 
The corresponding naphthalene diols might have made a better comparison (naphthalene being isomeric with azulene), but they would lack the $C_{2}$ symmetry of the azulene and benzene compounds, and neither the $\mathrm{CF}_{3}$ nor $\mathrm{CH}_{3}$ naphthalene diols have been reported. Thus, azulene enantiomers appear to exhibit better resolution than comparable phenyl compounds. It is likely that the azulene dipole moment enhances attraction of these molecules to the stationary phase, enforcing diastereomeric interactions more effectively and thus enhancing the enantioseparations.

Since four of these racemic azulene diols (3-6) represent two sets of diastereomers (RR/SS vs. SR/RS), we can compare the degree of resolution for diastereomeric pairs of enantiomers. On Chiralcel-OD-H, the enantiomers of the RR/SS diastereomers 3 and $\mathbf{5}$ averaged an $\mathrm{R}_{\mathrm{s}}$ of 3.4 and $\alpha$ of 2.4. In contrast, the RS/SR diastereomers 4 and 6 gave an average $R_{s}$ of 8.1 and $\alpha$ of 7.8. In both cases, the more polar (based on normal phase TLC mobility) RS/SR diastereomers were much better resolved than the less polar RR/SS diastereomers. This may be related to the polarities, as a rough correlation $\left(\mathrm{r}^{2}=0.65\right)$ exists between silica TLC $R_{f}$ and $R_{s}$ on Chiralcel OD-H. The earlier study of these compounds [13] revealed that the RS/SR diastereomers favor a conformation in which both $\mathrm{OH}$ groups are oriented toward the same face of the planar azulene system, which is the origin of the higher affinity for silica. Specifically how this might influence enantioselectivity is unclear. While some enantioseparations have been reported [17-19] for diastereomeric molecules, these studies have not attempted to correlate diastereomeric differences (e.g., polarity) with observed resolution. Thus, whether more polar diastereomers are generally better resolved than less polar ones is an open question. With the Lux cellulose 2 column, no large differences in resolution between diastereomers was evident.

The enantiomers of these compounds had not been separated prior to this work, and so there exists no comparison data. Characterization was complicated by the fact that traditional polarimetry at $589 \mathrm{~nm}$ was not possible for these purple compounds because of their strong absorption of yellow light. To avoid this problem, circular dichroism (CD) spectra were obtained on the separated enantiomers (Table 4).

Table 4. Summary of Circular Dichroism (CD) spectrum for each of the separated enantiomers. Each enantiomer is labeled by its order of elution from the HPLC. Retention times are given from the HPLC chromatograms obtained with the Chiralcel-OD-H column.

\begin{tabular}{|c|c|c|c|}
\hline & Compound & $\lambda(\mathrm{nm})$ & $\Delta \varepsilon$ \\
\hline \multirow[b]{2}{*}{1} & Faster $-1.43 \mathrm{~min}$ & 349.8 & 11.64 \\
\hline & Slower-1.61 min & 349.8 & -6.47 \\
\hline \multirow[b]{2}{*}{2} & Faster $-4.70 \mathrm{~min}$ & 356.3 & 11.23 \\
\hline & Slower $-9.80 \mathrm{~min}$ & 356.3 & -10.66 \\
\hline \multirow{2}{*}{3} & Faster $-1.75 \mathrm{~min}$ & 349.7 & -7.45 \\
\hline & Slower $-2.97 \mathrm{~min}$ & 349.7 & 8.60 \\
\hline \multirow[b]{2}{*}{4} & Faster $-1.30 \mathrm{~min}$ & 345.0 & -2.83 \\
\hline & Slower $-8.13 \mathrm{~min}$ & 345.0 & 4.17 \\
\hline \multirow[b]{2}{*}{5} & Faster $-1.88 \mathrm{~min}$ & 314 & -3.56 \\
\hline & Slower $-4.73 \mathrm{~min}$ & 314 & 7.12 \\
\hline \multirow{2}{*}{6} & Faster $-1.87 \mathrm{~min}$ & 339.7 & -9.67 \\
\hline & Slower- $-11.15 \mathrm{~min}$ & 339.7 & 10.70 \\
\hline
\end{tabular}


Each enantiomer was collected over several analytical runs and concentration was determined by UV spectroscopy. CD was run on each of the separated enantiomers, giving roughly equal but opposite curves of molar extinction versus wavelength, particularly in the region of 300-400 nm (Figure 6). However, lack of CD spectra of comparison compounds prevents identification of the configuration of the enantiomers.

Figure 6. CD spectrum for each of the separated enantiomers, 1-6.

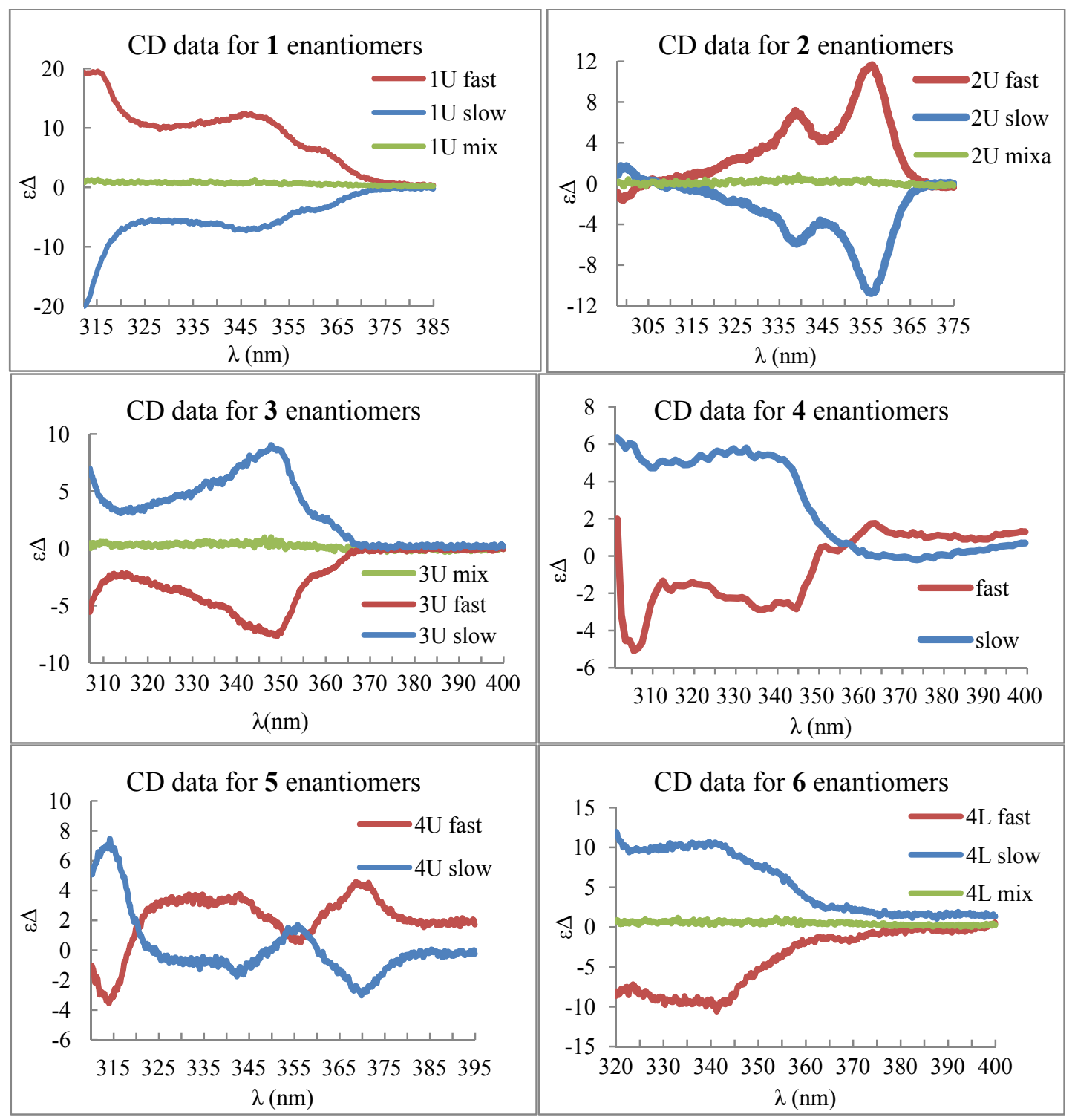

\section{Conclusions}

The enantioseparation of a series of azulene-centered 1,5-diols has been accomplished on two chiral HPLC columns, with Chiralcel OD-H generally being superior to the Lux cellulose 2 column. The azulene compounds were much better resolved than comparable phenyl compounds, suggesting that the polarity of the azulene ring is important in enforcing diastereomeric interactions with the stationary phase. In addition, an interesting diastereomer dependence on the enantioseparation was observed, with the more polar RS/SR diastereomers being much better resolved than the RR/SS enantiomers on Chiralcel OD-H. 


\section{Acknowledgments}

The authors would like to thank Phenomenex, Chiral Technologies and Regis Technologies for their chiral HPLC column screening process, which aided us in separating the enantiomers. We also thank the Robert A. Welch Foundation for partial support of this work (grant AA-1395).

\section{Authors Contributions}

Dana Horgen: Performed separations/measurements and analyzed data, Charles Garner: designed the study, analyzed data and wrote the manuscript.

\section{Conflicts of Interest}

The authors declare no conflict of interest.

\section{References}

1. Chiral Separations: Methods and Protocols (Methods in Molecular Biology Volume 243); Gübitz, G., Schmid, M.G., Eds.; Humana Press: Totowa, NJ, USA, 2004; pp. 1-424.

2. Principles of Asymmetric Synthesis, 2nd ed.; Gawley, R.A., Aubé, J., Eds.; Elsevier: Oxford, UK, 2012; pp. 76-79.

3. Dou, L.; Zeng, J.; Gerochi, D.; Duda, M.; Stuting, H. Chiral high-performance liquid chromatography methodology for quality control monitoring of dexfenfluramine. J. Chrom. A 1994, 679, 367-374.

4. Ribeiro, A.R.; Castro, P.M.L.; Tiritan, M.E. Chiral pharmaceuticals in the environment. Environ. Chem. Lett. 2012, 10, 239-253.

5. Anderson, A.G.; Strecker, B.M. Azulene VIII. A study of the absorption spectra and dipole moments of some 1- and 1,3-substituted azulenes. J. Am. Chem. Soc. 1959, 81, 4941-4946.

6. Liu, R.S.H. Colorful azulene and its equally colorful derivatives. J. Chem. Educ. 2002, 79, 183-185.

7. Zhang, L-Y.; Yang, F.; Shi, W-Q.; Zhang, P.; Li, Y.; Yin, S-F. Synthesis and antigastric ulcer activity of novel 5-isopropyl-3,8-dimethylazulene derivatives. Bioorgan. Med. Chem. Lett. 2011, $21,5722-5725$.

8. Okano, K. Synthesis and application of chiral hydrobenzoin. Tetrahedron 2011, 67, 2483-2572.

9. Naoshima, Y.; Kimura, T.; Mori, Y.; Kamezawa, M.; Tachibana, H.; Kohara, K.; Ohtani, T. The first synthesis of chiral azulene alcohol possessing a trifluoromethyl group by lipase-mediated biotransformation. Recent Res. Devel. Org. Bioorgan. Chem. 2004, 6, 1-9.

10. Redl, F.X.; Köthe, O.; Röckl, K.; Bauerr, W.; Daub, J.; Azulene appended cellulose: Synthesis, optical and chiroptical properties, film formation by electrochemical oxidation. Macromol. Chem. Phys. 2000, 201, 2091-2000.

11. Oki, M.; Nakamura, N. Restricted Rotation Involving the Tetrahedral Carbon. II. 2-Substituted 4,6,8-Trimethylazulenes. Bull. Chem. Soc. Jpn. 1971, 44, 1880-1885.

12. Anderson, A.G., Jr.; Anderson, R.G.; Hollander, G.T. Reactions of some 1-trihaloacetyl-8acetylazulenes with base. J. Org. Chem. 1965, 30, 131-138. 
13. Chen, A.H.; Yen, H.H.; Kuo, Y.C.; Chen, W.Z. Asymmetric synthesis and characterization of chiral 2,2'diamino-3,3'-diethoxycarbonyl-8-8'-diphenyl-1,1'-biazulene. Synth. Commun. 2007, 37, 2975-2987.

14. Horgen, D.A.; Klausmeyer, K.K.; Carson, C.; Shen, H.; Garner, C.M. Remarkably large remote-stereocenter induced chromatographic differences: Azulene 1,5-diols. Tetrahedron Lett. 2014, 55, 128-132.

15. Prakash, G.K.S.; Krishnamurti, R.; Olah, G.A. Synthetic methods and reactions. 141. Fluoride-induced trifluoromethylation of carbonyl compounds with trifluoromethyltrimethylsilane (TMS-CF 3 ). A trifluoromethide equivalent. J. Am. Chem. Soc. 1989, 111, 393-395.

16. Johnston, H.W.; Williams, J.L.R. Preparation of m-divinylbenzene. J. Am. Chem. Soc. 1947, 69, 2065-2065.

17. Yang, S.; Li, C.; Wang, S.; Zhao, L.; Hou, Z.; Lou, H.; Ren, D. Chiral separation of two diastereomeric pairs of enantiomers of novel alkaloid-ligand hybrids from Lobelia chinensis and determination of the tentative absolute configuration. J. Chrom. A 2013, 1311, 134-139.

18. Tabata, H.; Yoneda, T.; Oshitari, T.; Takahashi, H.; Natsugari, H. Stereochemistry of 1,5-Benzothiazepin-4-one S-Oxide: Insight into the Stereogenic Elements at the Sulfur Atom and Axis. J. Org. Chem. 2013, 78, 6264-6270.

19. Christov, P.P.; Hawkins, E.K.; Kett, N.R.; Rizzo, C.J. Simplified synthesis of individual stereoisomers of the 4-hydroxynonenal adducts of deoxyguanosine. Tetrahedron Lett. 2013, 54, 4289-4291.

(C) 2014 by the authors; licensee MDPI, Basel, Switzerland. This article is an open access article distributed under the terms and conditions of the Creative Commons Attribution license (http://creativecommons.org/licenses/by/3.0/). 